\title{
The quest for the grail: multidimensional efforts for understanding and targeting severe asthma
}

\author{
Mina Gaga ${ }^{1}$, Paul L.P. Brand ${ }^{2,3}$ and Neil C. Thomson ${ }^{4}$ \\ Affiliations: ${ }^{1}$ Athens Chest Hospital, 7th Respiratory Medicine Dept, Athens, Greece. ${ }^{2}$ Isala klinieken, Princess \\ Amalia Children's Clinic, Zwolle, The Netherlands. ${ }^{3}$ University Medical Centre, UMCG Postgraduate School of \\ Medicine, Groningen, The Netherlands. ${ }^{4}$ Institute of Infection, Immunity and Inflammation, University of \\ Glasgow, Glasgow, UK.
}

Correspondence: Mina Gaga, Athens Chest Hospital, 7th Respiratory Medicine Dept, 152 Mesogion Ave, Athens 11527, Greece. E-mail: minagagadyahoo.com

@ERSpublications

First results from the U-BIOPRED studies of adults and children with severe asthma http://ow.ly/RKJZ7

The exponential advance in biological and medical science, with the use of molecular and genetic testing as well as various "omics" approaches, has led to the elucidation of many disease pathways. It has also led to the identification of marked differences in the mechanisms, phenotypes and genotypes of diseases that were once thought to have similar characteristics and required similar therapies. An example of this approach is in cystic fibrosis, where patients with the specific G551D mutation respond extremely well to ivafactor (Kalydeco) [1]. Although this mutation is only found in about $5 \%$ of all cystic fibrosis patients, their response to treatment is so dramatic that it is truly life changing. However, the cost of this medication is extremely high and it is difficult to imagine that similar costs can be sustained in the long term for other, more common diseases. The number of available monoclonal antibodies and targeted drugs for severe or life-threatening disease, such as cancer and rheumatoid arthritis, is increasing rapidly and expected to continue to rise in the next decade $[2,3]$. Moreover, we are now faced with the severe end of the spectrum of many diseases, in which the clinical characteristics and response to treatment differ considerably from mild disease.

Severe, difficult-to-treat asthma is an example of this. Although asthma is a very common disease and although the majority of patients respond well to low-dose controller medications such as inhaled corticosteroids and long-acting $\beta$-agonists, a subset of patients remain uncontrolled despite the use of high-dose multiple-drug daily controller therapy. These patients experience substantial morbidity due to the disease and to the adverse effects of high-dose corticosteroids [4-7] and generate high healthcare costs. The prevalence of such severe, difficult-to-treat asthma is difficult to assess because remediable factors such as non-adherence, incorrect inhalation technique and comorbidities play a role in many patients referred to specialist care because of uncontrolled asthma despite the use of daily controller therapy [4]. After addressing these factors in adults, $\sim 3-10 \%$ of patients are estimated to have severe refractory asthma [4-6]. In children, such studies are rare. In an English multicentre study of asthmatic children with uncontrolled asthma despite inhaled corticosteroid use, only $7 \%$ of eligible patients remained uncontrolled after addressing the basics of asthma management [8]. In a recent Dutch study, only $3 \%$ of patients referred for problematic severe asthma fulfilled the criteria for true therapy-resistant asthma [9]. Unfortunately, treatment options are limited for severe asthmatics that remain uncontrolled despite maximal standard therapy. Omalizumab has been used successfully both in children and in adults, and bronchial thermoplasty is an option in adults with therapy-resistant severe asthma [10-12]. A humanised monoclonal antibody to human interleukin-5, mepolizumab, was recently approved by the US Food and

Received: Aug 262015 | Accepted: Aug 262015

Conflict of interest: None declared.

Copyright OERS 2015 
Drug Administration for add-on maintenance treatment in patients aged $\geqslant 18$ years with severe eosinophilic asthma [13]. Despite these developments, there is a lack of understanding of severe asthma mechanisms, limiting the possibilities to develop novel treatments for severe asthma [14].

It is becoming increasingly apparent that severe asthma may be due to many different mechanisms and that various subsets of severe asthma patients should be clustered into specific endotypes, i.e. groups with similar clinical and pathophysiological characteristics $[4,15,16]$. This is particularly true in adults, where the increased understanding of the multidimensional nature of severe asthma has allowed the development of new targeted treatments, such as monoclonal antibodies targeting IgE in allergic patients [10], targeting persistent eosinophilic inflammation and targeting T-helper cell type 2 (Th2)-high inflammation [13, 17, 18], and has allowed the search for biomarkers that predict a beneficial response to these treatments [19].

Although it is assumed that this also applies to severe asthma in children, the limited number of studies evaluating children and adolescents with severe asthma limits the possibilities for paediatricians to understand and treat this condition even further $[9,20]$.

Identifying specific clusters of patients with severe/difficult-to-treat disease, researching and developing medications for them and monitoring the course of the disease and the response to treatment, requires effort, expertise and resources. It also requires concerted action. Clinicians, researchers and patients, and also the pharmaceutical industry and health policy makers, should get together, identify the pressing needs and the research questions, design a robust research methodology and provide the technical and financial resources.

Over the last 15 years, several international collaborative consortia have investigated the pathogenetic mechanisms of severe asthma [4, 21, 22]. The European Network For Understanding Mechanisms Of Severe Asthma (ENFUMOSA) conducted a cross-sectional study to characterise the clinical and selective inflammatory variables in 163 adults with severe asthma compared with 158 subjects with well-controlled asthma [21]. In this study, patients with severe asthma were more often female, had worse asthma control and less atopy, and higher sputum neutrophil counts than the well-controlled group [21]. The National Heart, Lung, and Blood Institute's Severe Asthma Research Program (SARP) undertook a comprehensive phenotypic characterisation of over 583 adults with severe asthma and people with mild and moderate asthma as well as 300 children with asthma, recruited mainly from academic sites in the USA [7, 22]. In a series of publications, the SARP studies have provided unique insights into the heterogeneous nature of the inflammatory and structural abnormalities associated with severe asthma and have identified clusters differing in characteristics such as age of onset, body mass index, sex and fixed airway obstruction [7]. The Longitudinal Assessment of Clinical Course and BIOmarkers in Severe Chronic AIRway Disease (BIOAIR) study compared phenotypes defined either by biomarkers or by physiological variables in 93 adults with severe asthma and 76 adults with mild-to-moderate asthma. Phenotypes determined by sputum cell counts were less stable than those defined by physiological variables, especially in severe asthma [23].

The U-BIOPRED project

The most recent international collaborative project in severe asthma is the Unbiased Biomarkers for the Prediction of Respiratory Disease Outcomes (U-BIOPRED) study. This project is designed to identify new phenotypes/endotypes and treatment targets using "omics" technologies (transcriptomic, proteomic, lipidomic and metabolomic) [24] and applying a systems biology approach. The project involves the integration of data from patient-reported outcomes, invasive (bronchial biopsies in adults) and noninvasive (blood, sputum, urine and exhaled air) samples. It is hoped that this methodology will identify distinct phenotypic handprints of severe asthma and result in the unbiased discovery of new treatments for both adults and children with severe asthma. The project commenced in 2009 within the framework of the European Union Innovative Medicines Initiative (IMI) and the consortium involves partnerships between 20 academic institutions, 11 pharmaceutical companies and six patient groups.

Two initial articles from this study are published in this issue of the European Respiratory Journal (ERJ), one referring to adult patients [25] and one to children [26].

The first manuscript describes the clinical, physiological and inflammatory features of the adult participants recruited to the study and as such is a key publication for the interpretation of future reports on the mechanisms of disease identified using new technologies. A systematic algorithmic approach developed by the consortium was used to evaluate patients presenting with chronic severe asthma symptoms and to identify those subjects with severe refractory asthma [27].

SHAW et al. [25] report the results of a cross-sectional study of clinical outcomes and inflammatory biomarkers from adults with severe asthma (nonsmokers $\mathrm{n}=311$ and smokers/ex-smokers $\mathrm{n}=110$ ), mild/ moderate asthma $(n=88)$ and healthy controls $(n=101)$ recruited from 11 European countries. A major strength of the study is the recruitment of a large cohort of adults with severe refractory asthma. 
The main findings were that patients with severe asthma had worse symptoms and more exacerbations, despite high-dose treatment including oral corticosteroids in $45 \%$ of participants. Severe asthma patients also had higher levels of anxiety and depression as well as a higher incidence of comorbidities of nasal polyps and gastro-oesophageal reflux compared with patients with mild/moderate asthma. Lung function was lower and sputum eosinophils higher in the severe group despite receiving more treatment. The clinical features in this U-BIOPRED severe adult asthma cohort are in general similar to the SARP and ENFUMOSA cohorts. The U-BIOPRED severe asthma cohort has a slightly higher exacerbation rate $(2.5$ per year), lower forced expiratory volume in $1 \mathrm{~s}$ (68\% predicted) and a higher number of patients on oral corticosteroids than the SARP and ENFUMOSA cohorts. Eosinophilic airway inflammation was present in a similar proportion of patients.

The inclusion of current smokers with severe asthma $(n=42)$ as well as ex-smokers with severe asthma with a smoking history of $>5$ pack-years $(n=68)$ in the U-BIOPRED cohort is important, since both groups were excluded from the SARP and ENFUMOSA cohorts. Adult smokers with asthma, including those with severe diseases, have poor symptom control, increased exacerbation rates and high levels of healthcare utilisation as well as an attenuated therapeutic response to corticosteroids compared with never-smokers with asthma [28-30]. Current smokers and ex-smokers (smoking history $>10$ pack-years) are generally excluded from clinical trials in asthma despite a prevalence of $20-30 \%$ active smoking in the general asthmatic population and $\sim 10 \%$ in severe asthma [28].

It is reassuring to note that the baseline characteristics of the U-BIOPRED cohort are similar to patients included in national registries of severe asthma such as the British Thoracic Society Severe Asthma Registry [28, 31] and Belgian Severe Asthma Registries [32, 33], which suggests that findings from U-BIOPRED should be generalisable to "real-life" patients with severe asthma.

Although both interesting and important, the study by SHAw et al. [25] has some limitations. First, the classification used to define subgroups of severe asthma based on smoking status may obscure important differences in clinical outcomes and inflammatory mechanisms. The adults with severe asthma were classified into two groups, a nonsmoker subgroup who had a smoking history of $<5$ pack-years and a second subgroup that combined current smokers $(n=42)$ and ex-smokers with a smoking history of $>5$ pack-years $(n=68)$. Although data on the clinical, physiological and inflammatory variables in current smokers with asthma compared with ex-smokers with asthma is limited, it does suggest that these two groups differ [28, 34, 35]. An alternative classification of never-smokers, ex-smokers (subdivided into different pack-year histories) and current smokers may provide greater insight into possible mechanisms in asthma and in particular those that are related or unrelated to current or previous cigarette smoking. Nevertheless, the criteria used to classify the severe asthma subgroups in U-BIOPRED may be less important when the unbiased analyses of the dataset are performed. Secondly, there was a relatively low number of samples collected for some biological measurements, which may influence the generalisability of the findings from the new technologies. For example, adequate sputum was obtained in only $42-50 \%$ of participants and bronchoscopy was performed in only $21 \%$ of nonsmokers with severe asthma, $8 \%$ of smokers and ex-smokers with severe asthma and $45 \%$ of nonsmokers with mild/moderate asthma. The difference in the number of bronchoscopy samples obtained in each group could influence the validity of the results of comparisons between groups in inflammatory variables.

The second article is a cross-sectional analysis of baseline data from the paediatric U-BIOPRED project, in which cohorts of children with severe asthma and severe preschool wheeze are compared with those with mild/moderate asthma and preschool wheeze. The international approach, the combination of school-aged and preschool cohorts, and the extensive standardised description of the cohorts are major strengths of this paper. Children were recruited in seven paediatric and paediatric respiratory units throughout Europe. Comparisons between the cohorts with severe disease and those with mild/moderate disease showed highly significant differences in symptom burden, exacerbation frequency, Asthma Control Test results and (patient's or parent's) quality of life. This is not surprising as these differences define the distinction between mild/moderate and severe disease. Much more fascinatingly, there were hardly any differences in demographic, clinical, lung function and inflammatory characteristics between the severe and mild/moderate cohorts. This is clearly different from the situation in adults, where severe asthma is distinguishable from mild/moderate asthma not only in terms of symptoms, but also in physiological and inflammatory characteristics. Why, then, do children with severe asthma/wheeze have so many more symptoms and exacerbations and a poorer quality of life than children with mild/moderate disease? Although the "omics" data from the paediatric U-BIOPRED study may shed more light on this issue, we should also explore alternative areas of explanation, such as psychological issues and family structure and functioning.

In the paediatric U-BIOPRED study published in this issue of the ERJ, objective evidence of exposure to tobacco smoke at home was the only putative determinant examined that was more common in preschool 
children with severe wheeze than in those with mild/moderate wheeze. These differences between children and adults in the characteristics of severe asthma call for serious caution in extrapolating data from studies in adults to the situation in children. Drugs that work in adults with severe asthma may be utterly useless in children with such severe disease, for example. Another interesting finding in the paediatric U-BIOPRED paper was that comorbidities such as food allergy and gastro-oesophageal reflux, although frequently reported by parents, were hardly ever confirmed by appropriate testing. This highlights the difficulties in charting the prevalence and importance of comorbidities in children with asthma [35]. The impact of comorbidities of childhood asthma on the severity and control of the disease is an area of research that has only recently begun to expand. The impact of allergic rhinitis on asthma control has been established $[36,37$ ] and it is likely that treating allergic rhinitis in children may help to control their asthma [19, 36-38]. The effects of other comorbidities on asthma control and severity in children are poorly studied, however.

The differences between the findings of the adult and paediatric U-BIOPRED studies are fascinating. Whilst the pathophysiological mechanisms of the different phenotypes of severe asthma in adults are becoming increasingly clear, the results of the paediatric U-BIOPRED study confirm earlier observations that these mechanisms in paediatric severe asthma remain largely elusive. This is partly due to ethical restraints limiting the possibility of performing invasive diagnostic procedures in children, such as bronchoscopy to obtain bronchial biopsies or perform bronchoalveolar lavage. The results of the "omics" analyses of the paediatric U-BIOPRED study are therefore eagerly awaited.

In conclusion, the U-BIOPRED investigators have successfully recruited a large cohort of adults and children with severe asthma and collected a wide range of biological samples from these patients and controls, which is a major achievement. The application of unsupervised analyses of the clinical variables and "omics" datasets will hopefully help define distinct phenotypes and endotypes of severe asthma and identify new treatments. Communication of the results of these analyses is eagerly anticipated by the scientific community and by patients with severe asthma who currently have limited effective treatment options to alleviate their poorly controlled symptoms.

The U-BIOPRED studies are unique in their multidisciplinary collaboration, bringing together not only researchers and clinicians, but also pharmaceutical industries and patient organisations. Clearly, this is the way forward in our endeavours to understand disease mechanisms of severe asthma better, to be able to provide effective solutions and medications to help patients with more severe disease. This collaborative approach may serve as a model for similar studies in other chronic or life-threatening diseases. Understanding disease mechanisms better is only one part of the equation leading to better treatment options. Establishing good communication among all interested parties, considering all the important questions and setting specific goals are other steps, and they must be addressed in constructive collaboration between clinicians, researchers, patients, commercial parties and healthcare policy makers. We have to think big but tread carefully, meticulously classifying disease characteristics and providing insight, knowledge and solutions that are applicable and that can be adopted in everyday clinical practice. And we need to discuss the cost with all interested parties so that all solutions can be worth pursuing but also financially sustainable in the long term.

\section{References}

1 Accurso FJ, Rowe SM, Clancy JP, et al. Effect of VX-770 in persons with cystic fibrosis and the G551D-CFTR mutation. N Engl J Med 2010; 363: 1991-2003.

2 Rosell R, Karachaliou N. Lung cancer in 2014: optimizing lung cancer treatment approaches. Nat Rev Clin Oncol 2015; 12: 75-76.

3 López-González R, León L, Loza E, et al. Adherence to biologic therapies and associated factors in rheumatoid arthritis, spondyloarthritis and psoriatic arthritis: a systematic literature review. Clin Exp Rheumatol 2015; 33: 559-569.

4 Chung KF, Wenzel SE, Brozek JL, et al. International ERS/ATS guidelines on definition, evaluation and treatment of severe asthma. Eur Respir J 2014; 43: 343-373.

5 Global Initiative for Asthma. Global Strategy for Asthma Management and Prevention. 2015. Available from www.ginasthma.org

6 Hekking PP, Wener RR, Amelink M, et al. The prevalence of severe refractory asthma. J Allergy Clin Immunol 2015; 135: 896-902.

7 Jarjour NN, Erzurum SC, Bleecker ER, et al. Severe asthma: lessons learned from the National Heart, Lung, and Blood Institute Severe Asthma Research Program. Am J Respir Crit Care Med 2012; 185: 356-362.

8 Lenney W, McKay AJ, Tudur Smith C, et al. Management of Asthma in School age Children On Therapy (MASCOT): a randomised, double-blind, placebo-controlled, parallel study of efficacy and safety. Health Technol Assess 2013; 17: 1-218.

9 de Groot EP, Kreggemeijer WJ, Brand PL. Getting the basics right resolves most cases of uncontrolled and problematic asthma. Acta Paediatr 2015; 104: 916-921.

10 Hanania NA, Alpan O, Hamilos DL, et al. Omalizumab in severe allergic asthma inadequately controlled with standard therapy: a randomized trial. Ann Intern Med 2011; 154: 573-582. 
11 Deschildre A, Marguet C, Salleron J, et al. Add-on omalizumab in children with severe allergic asthma: a 1-year real life survey. Eur Respir J 2013; 42: 1224-1233.

12 Castro M, Rubin AS, Laviolette M, et al. Effectiveness and safety of bronchial thermoplasty in the treatment of severe asthma: a multicenter, randomized, double-blind, sham-controlled clinical trial. Am J Respir Crit Care Med 2010; 181: 116-124.

13 Fajt ML, Wenzel SE. Asthma phenotypes and the use of biologic medications in asthma and allergic disease: the next steps toward personalized care. J Allergy Clin Immunol 2015; 135: 299-310.

14 Barnes PJ, Bonini S, Seeger W, et al. Barriers to new drug development in respiratory disease. Eur Respir J 2015; 45: 1197-1207.

15 Lötvall J, Akdis CA, Bacharier LB, et al. Asthma endotypes: a new approach to classification of disease entities within the asthma syndrome. J Allergy Clin Immunol 2011; 127: 355-360.

16 Fahy JV. Type 2 inflammation in asthma - present in most, absent in many. Nat Rev Immunol 2015; 15: 57-65.

17 Ortega HG, Liu MC, Pavord ID, et al. Mepolizumab treatment in patients with severe eosinophilic asthma. $N$ Engl J Med 2014; 371: 1198-1207.

18 Ortega H, Chupp G, Bardin P, et al. The role of mepolizumab in atopic and nonatopic severe asthma with persistent eosinophilia. Eur Respir J 2014; 44: 239-241.

19 Arron JR, Choy DF, Scheerens H, et al. Noninvasive biomarkers that predict treatment benefit from biologic therapies in asthma. Ann Am Thorac Soc 2013; 10: Suppl., S206-S213.

20 Bracken M, Fleming L, Hall P, et al. The importance of nurse-led home visits in the assessment of children with problematic asthma. Arch Dis Child 2009; 94: 780-784.

21 The ENFUMOSA Study Group. The ENFUMOSA cross-sectional European multicentre study of the clinical phenotype of chronic severe asthma. Eur Respir J 2003; 22: 470-477.

22 Fitzpatrick AM, Teague WG, Meyers DA, et al. Heterogeneity of severe asthma in childhood: confirmation by cluster analysis of children in the National Institutes of Health/National Heart, Lung, and Blood Institute Severe Asthma Research Program. J Allergy Clin Immunol 2011; 127: 382-389.

23 Kupczyk M, Dahlén B, Sterk PJ, et al. Stability of phenotypes defined by physiological variables and biomarkers in adults with asthma. Allergy 2014; 69: 1198-1204.

24 Wheelock CE, Goss VM, Balgoma D, et al. Application of 'omics technologies to biomarker discovery in inflammatory lung diseases. Eur Respir J 2013; 42: 802-825.

25 Shaw DE, Sousa AR, Fowler SJ, et al. Clinical and inflammatory characteristics of the European U-BIOPRED adult severe asthma cohort. Eur Respir J 2015; 46: 1308-1321.

26 Fleming L, Murray C, Bansal AT, et al. The burden of severe asthma in childhood and adolescence: results from the paediatric U-BIOPRED cohorts. Eur Respir J 2015; 46: 1322-1333.

27 Bel EH, Sousa A, Fleming L, et al. Diagnosis and definition of severe refractory asthma: an international consensus statement from the Innovative Medicine Initiative (IMI). Thorax 2011; 66: 910-917.

28 Thomson NC, Chaudhuri R, Heaney LG, et al. Clinical outcomes and inflammatory biomarkers in current smokers and exsmokers with severe asthma. J Allergy Clin Immunol 2013; 131: 1008-1016.

29 Thomson NC, Chaudhuri R. Asthma in smokers: challenges and opportunities. Curr Opin Pulm Med 2009; 15 $39-45$.

30 Polosa R, Thomson NC. Smoking and asthma: dangerous liaisons. Eur Respir J 2013; 41: 716-726.

31 Heaney LG, Brightling CE, Menzies-Gow A, et al. Refractory asthma in the UK: cross-sectional findings from a UK multicentre registry. Thorax 2010; 65: 787-794

32 Schleich F, Brusselle G, Louis R, et al. Heterogeneity of phenotypes in severe asthmatics. The Belgian Severe Asthma Registry (BSAR). Respir Med 2014; 108: 1723-1732.

33 Schleich FN, Chevremont A, Paulus V, et al. Importance of concomitant local and systemic eosinophilia in uncontrolled asthma. Eur Respir J 2014; 44: 97-108.

34 Clatworthy J, Price D, Ryan D, et al. The value of self-report assessment of adherence, rhinitis and smoking in relation to asthma control. Prim Care Respir J 2009; 18: 300-305.

35 Broekema M, ten Hacken NH, Volbeda F, et al. Airway epithelial changes in smokers but not in ex-smokers with asthma. Am J Respir Crit Care Med 2009; 180: 1170-1178.

36 de Groot EP, Duiverman EJ, Brand PL. Comorbidities of asthma during childhood: possibly important, yet poorly studied. Eur Respir J 2010; 36: 671-678.

37 de Groot EP, Nijkamp A, Duiverman EJ, et al. Allergic rhinitis is associated with poor asthma control in children with asthma. Thorax 2012; 67: 582-587.

38 Deliu M, Belgrave D, Simpson A, et al. Impact of rhinitis on asthma severity in school-age children. Allergy 2014; 69: 1515-1521. 